\title{
Hitos de la investigación en la Escuela Nacional de Salud Pública de Cuba, 1977-2010
}

\section{Landmarks of research in the National School of Public Health, 1977-2010}

\section{DraC. Nereida Rojo Pérez, MsC. Carmen Valenti Pérez}

Escuela Nacional de Salud Pública. La Habana, Cuba.

La Escuela Nacional de Salud Pública en el año 2010 cierra un productivo ciclo de trabajo en el campo de la investigación-desarrollo, lo que significa nuevos retos en el camino a seguir, ya que la condición de Excelencia otorgada a la institución por el Sindicato Nacional de Trabajadores de la Salud y el Ministerio de Salud Pública (MINSAP) es, ante todo, un compromiso para contribuir a los desafíos en esta área.

La Escuela Nacional de Salud Pública tiene como referentes en el campo de la investigación al Instituto de Desarrollo de la Salud (IDS) formado a partir de un núcleo de salubristas de la Dirección Nacional de Estadísticas (DNE) del Ministerio de Salud Pública (MINSAP), quienes en la década de los sesenta del siglo xx, iniciaron y dieron continuidad a investigaciones transcendentes para el desarrollo de la salud pública cubana. ${ }^{1}$ Entre estas investigaciones se pueden citar: las encuestas nacionales de fecundidad en Cuba, los estudios de crecimiento y desarrollo humano, la investigación de mortalidad perinatal, el estudio de los componentes y determinantes del estado de salud de la población, así como aquellos relacionados con la planificación y organización de los servicios de salud, los estudios de costo, la formación de los recursos humanos para el sector, lo que posibilitó que se incrementaran y fortalecieran las interrelaciones entre instituciones académicas y científicas tanto nacionales como internacionales.

En los ochenta, esta institución se convierte en la Facultad de Salud Pública (FSP) y sus grupos de investigadores van a fortalecer el trabajo en otras áreas $\mathrm{e}$ instituciones del MINSAP. 
La FSP fue creada para responder a la demanda incrementada de la formación docente del cuarto nivel de enseñanza (posgrado) en el campo de las disciplinas propias de la salud pública que cubría los dos subsistemas de este nivel: la capacitación profesional con los cursos, diplomados y especialidades y la formación académica con las maestrías y doctorados. Este tipo de formación indisolublemente vinculada a la práctica social y su transformación, llevó a la necesidad de identificar problemas prioritarios de investigación, tanto nacionales como internacionales, y a generar grupos de trabajo para desarrollar y dar respuesta a los diferentes temas que se abordaban en las tesis de grados en relación con los problemas de salud y sus respuestas en el ámbito social y sanitario.

Surgen así, los grupos para el estudio de los problemas relacionados con el análisis de la situación de salud y su aplicación en la práctica sanitaria, el de familia y comunidad, el de capacitación a equipos de dirección, el de condiciones de vida, de vigilancia en salud, de evaluación de programas, sistemas y servicios de salud, de planificación estratégica y de gestión en salud, este último radicaba en el Centro de Perfeccionamiento Gerencial adscripto a la FSP desde su creación en 1998. La preparación de grupos en estas materias específicas y la obtención de resultados, a partir de las tesis de grado, posibilitaron las condiciones para el surgimiento en 1996 del Programa Ramal de Investigaciones en Sistemas y Servicios de Salud.

Sin embargo, no es hasta el año 2000, en que se fusionan la FSP y el Centro Nacional de Perfeccionamiento Médico (CENAPEM) como Escuela Nacional de Salud Pública, se aprueba la planificación estratégica del área de investigación-desarrollo y se fijan los objetivos y prioridades de la investigación a nivel institucional.

En el marco del programa ramal se amplió la visión temática y el alcance territorial de la investigación en el campo, centrada ahora en la eficiencia de los servicios y las políticas de salud, especialmente los estudios de evaluación económica, de calidad de la atención, del componente promocional y preventivo de los programas y servicios, así como de los determinantes sociales de la salud, ${ }^{2}$ de indagación acerca de nuevas formas e indicadores para la medición de la mortalidad, la morbilidad y el uso adecuado de los niveles de atención, el sistema de salud, su historia, principios, conceptualizaciones, los modelos organizacionales de control y vigilancia en salud, entre otros. También sería importante señalar que la investigación en sí misma, constituyó un tema de interés en la búsqueda de modelos más costo-efectivos de abordaje de la realidad y la aproximación hacia un modelo heurístico y hermenéutico que permitiera una mejor comprensión desde lo biosocial de los grupos poblacionales más vulnerables, como la mujer, el niño, los ancianos, las personas con conductas sexuales de riesgo o enfermedades crónicas y determinadas regiones o zonas geográficas.

En el balance realizado al cierre del decenio que acaba de concluir, la Escuela Nacional de Salud Pública había cumplido con las políticas y metas trazadas en la planificación estratégica con la lamentable excepción de los indicadores de publicaciones (Castell P, Rojo N, Morales I. Frontline experiences in Graduate Programs Cuba's National School of Public Health. International Conference on Public Health Education in Low and Middle Income Countries. Processes, Proceedings and Proposed New Steps. Public Health Foundation of India. 12-14 de agosto de 2008).

La gestión eficiente del Programa Ramal de Investigaciones en Sistemas y Servicios de Salud, permite obtener resultados e identificar experiencias de avanzadas que pueden ser aplicadas en otros programas similares. Por la importancia de los resultados alcanzados se instaura en la convocatoria del año 2009 del Premio Anual de la Salud, la categoría de Investigaciones en Sistemas y Servicios de Salud. 
A partir del incremento en la presentación de proyectos para el Programa Ramal se realiza el diseño, se elabora la propuesta y se aprueba por las autoridades competentes, el Programa Ramal de Gestión y Formación de Recursos Humanos para la Salud, el que ya ha efectuado dos convocatorias con proyectos de todo el país.

Otros resultados a destacar son que más del $30 \%$ de los profesores están trabajando en proyectos de investigación aprobados en algún programa ramal y de ellos, hay 22 proyectos en el Programa Ramal de Investigaciones en Sistemas y Servicios de Salud. Al cierre del 2010 la institución contaba con cinco proyectos en ejecución financiados por organismos internacionales con resultados científicos acreditados.

El Comité de Ética de la Investigación fue creado y conjuntamente con el Consejo Científico han impulsado el desarrollo de investigaciones de calidad y pertinencia al Sistema Nacional de Salud y a la sociedad cubana.

Se han obtenido 12 registros del Centro de Derecho de Autor (CENDA) y varios premios en los concursos a los diferentes niveles del Premio Anual de la Salud y premios de Mejores Tesis de Grado Científico, en la sección de Biomedicina de la Comisión Nacional de Grados Científicos.

El trabajo en red ha sido fomentado, ejemplo de ello son la red de Investigaciones en Sistemas y Servicios en Salud, la Red de Enfermería en Salud Infantil (ENSI/Cuba), la Red Iberoamericana de Epidemiología y Sistemas de Información en Cáncer, entre otras.

Resultados en temas prioritarios para la salud pública cubana han sido acreditados por áreas e instituciones competentes del MINSAP y otros organismos del Estado, entre ellas:

- Enfermedades no transmisibles y otros daños.

- Historia de la Salud Pública.

- Gestión y formación de recursos humanos.

- Metodologías para estudios de determinantes del estado de salud.

- Metodologías para el estudio de programas y de los servicios de salud.

- Economía de la Salud.

- Salud Pública Internacional.

- Medicina Social.

Las relaciones de cooperación, a través de proyectos, se han mantenido desde hace más de dos décadas con organismos del Sistema de Naciones Unidas y otros financiadores externos, entre los principales: el Fondo de Naciones Unidas para Actividades de Población, el Fondo Mundial de Lucha contra el VIH/sida, la tuberculosis y la malaria, el Fondo de Naciones Unidas para la Infancia y el Athlantic Charitable Trust. 
No obstante los logros, el mayor desafío contemporáneo para la Escuela Nacional de Salud Pública, definido por las autoridades institucionales, es acompañar de manera eficiente al Sistema Nacional de Salud en la implementación y validación de estrategias sanitarias encaminadas al incremento de la cobertura, la accesibilidad, el logro de la equidad, la calidad de los servicios y al desarrollo de los valores en los trabajadores de la salud, donde la Atención Primaria de la Salud constituye el eslabón fundamental.

La Escuela Nacional de Salud Pública diseñó y puso en marcha una estrategia para alcanzar tales desafíos, que se resume en los siguientes objetivos de trabajo:

- Promover la excelencia institucional a través del cambio en la concepción, el lugar y la trascendencia de la investigación.

- Aprovechar todos los espacios para la gestión de la investigación, la comunicación y aplicación de los resultados de forma eficiente y sistemática

- Realizar una gestión eficiente de los programas ramales para producir investigaciones de alta calidad, en territorios, programas, servicios e instituciones, que permita a los directivos basar sus decisiones en resultados científicos.

- Concentrar los recursos y potencialidades de la Escuela Nacional de Salud Pública en el desarrollo de investigaciones en las líneas de acción estratégicas definidas para el periodo 2010-2014.

- Promover alianzas con centros afines, nacionales y extranjeros, dirigidas al intercambio científico, la colaboración técnica y la búsqueda de oportunidades de financiamiento.

En el último Pleno de la Academia de Ciencias de Cuba en noviembre de 2010, se hizo un llamado al papel de los científicos ante la actual situación económica y social que vive Cuba y se subrayó la importancia de la ciencia como pilar de innovación tecnológica indispensable para el desarrollo así como la necesidad de estimular el pensamiento, los hábitos y las habilidades científicas para responder a los problemas del futuro.

Sirva este recuento también para reconocer el trabajo de salubristas anónimos y profesores de la Escuela Nacional de Salud Pública que nos precedieron, algunos ya no están con nosotros, y otros que nos acompañan en el empeño de hacer de la investigación biosocial y sociosanitaria el mejor argumento para la formulación, revisión e implementación de nuevas políticas.

\section{REFERENCI AS BI BLI OGRÁFI CAS}

1. López Espinosa JA, Díaz del Campo S. La Revista Cubana de Salud Pública en tres décadas de circulación (1975-2004). Rev Cubana Salud Pública [Internet]. 2005 [citado 14 Nov 2011]; 31(1). Disponible en:

http://scielo.sld.cu/scielo.php?script=sci_arttext\&pid=S0864-

$\underline{34662005000100001 \& \operatorname{lng}=\mathrm{es} \& \mathrm{nrm}=\mathrm{iso \& t} \operatorname{lng}=\mathrm{es}}$ 
2. Rojo Pérez N. Investigaciones en Sistemas y Servicios de Salud en Cuba y su proyección hasta el 2015. Rev Cubana Salud Pública [Internet]. 2010 [citado 14 Nov 2011];36(3). Disponible en:

http://scielo.sld.cu/scielo.php?script $=$ sci arttext\&pid $=$ S0864-

34662010000300004\&lng=es\&nrm=iso\&tIng=es

Recibido: 8 de marzo de 2011.

Aprobado: 11 de octubre de 2011.

Nereida Rojo Pérez. Escuela Nacional de Salud Pública. Calle 100 No. 1132 e/ E y Perla. Altahabana, Municipio Boyeros. La Habana, Cuba.

Correo electrónico: nereida.rojo@infomed.sld.cu 